\title{
College Students Psychological Features and Intervention Strategies under Sudden Crisis -Analysis Based on COVID-19 Epidemic Situation
}

\author{
Chunlei Zhang \\ Heilongjiang Bayi Agricultural University, Heilongjiang Daqing, 163319, China
}

Keywords: COVID-19, college students, emergencies, psychological counseling

\begin{abstract}
Since the application of psychological intervention methods in the prevention and treatment of COVID-19, the higher education system has paid more and more attention to the psychological conditions of college students. The prevention and treatment of COVID-19 is also constantly advancing, and the application of psychological intervention in it has also been continuously improved and achieved. Although the good results displayed, but there are still many deficiencies in this process. This article combs the current situation of COVID-19 psychological intervention work carried out by universities or colleges by referring to relevant materials and actual cases among the college students and proposes countermeasures for how to strengthen psychological intervention work in the prevention of pandemic on campus.
\end{abstract}

\section{Introduction}

With the development of the disease, COVID-19 virus-infected patients and patient will produce a variety of complex psychological problems and obstacles, which are mainly manifested in the indifferent to the crisis, the state of transition and the state of incompetence, and thus will produce a series of physiological and psychological. The response, these psychological reactions and mental disorders will also vary according to personal cultural education, occupation, economic situation, infection background, disease stage, etc. All of universities or colleges around China have adopted various ways and taken timely actions preventing students from psychological problems faced nowadays.

\section{Psychological problems existing in COVID-19 infected patients and patients}

a). Difficult to face inspection results

The first reaction of COVID-19 virus infected people and patients when they first learned that they had COVID-19 was shock and denial, evading reality. This is because COVID-19 is difficult to cure and subject to external discrimination. Leading to discrimination and family involvement, if long-term to maintain the attitude of denial and avoidance, COVID-19 virus infected persons and patients may refuse to accept their due social responsibilities.

b). Numbness and indulgence in life

College students with COVID-19 infection and patients may also have a psychological reaction 
to resentment and feel unlucky, and they and their families must be under tremendous physical, psychological, and economic pressure. They will also suffer from social discrimination, and they will not be able to receive assistance. Prevention and control measures are not in place, giving COVID-19 virus infected people and patients that they are marginalized by social isolation, lack of sense of social belonging, dissatisfaction and hatred of society, and even abnormal psychology of revenge for society. It is a dangerous signal. If it cannot be guided in time and correctly, it will lead to defamation, insults, human rights violations, violations of law and discipline, etc., which is not conducive to social stability and unity. Some infected college students will also show numbness and enjoyment psychology, these psychologies are the flow of COVID-19.

\section{c). Suspicious and indifferent in interpersonal communication}

Students infected with COVID-19 virus and patients suffer from double pressures from themselves and from the outside, becoming sensitive and suspicious, often because of the words, actions, expressions or eyes of others will make them feel that others are discussing him. Disease, jokes, discriminates against him, and isolates him. Because of all kinds of fears and fears, they are alienated from groups and families in behavior, but they are psychologically eager to care, help, and understand. In various contradictions and pressures, COVID-19 virus infected students will have many extreme emotional and psychological problems. If psychological intervention is not carried out in time, it will increase social unrest and increase the crime rate, which will eventually make COVID-19 The occurrence of the opposition between the virus-infected students and the general public, therefore, it is very necessary to psychologically intervene in the COVID-19 virus-infected person and patient, and it deserves the attention of relevant departments.

Social discrimination has led to the reluctance of COVID-19 virus-infected students to face the society and the public positively, hiding their illness, making them unwilling to seek help and treatment from relevant organizations, leading to delays and worsening of their illness. Take a negative attitude towards their condition. Psychological problems such as inferiority complex, self-blame, depression, and irritability make them out of the group, lonely, interfere with others' disease and behavior, and interfere with the normal work of the intervention. Psychological intervention of COVID-19 virus-infected college students can help them alleviate the psychological problems caused by their living environment and their own pressure, so that they can face their illness with a positive attitude, eliminate self-discrimination, and promote the doctor-patient The establishment of good relationships between nurses, between infected persons, and between infected persons and their families, their positive attitudes also help to eliminate social discrimination, so that the prevention and treatment of COVID-19 can be smoother get on.

\section{Actively respond to COVID-19 prevention measures}

a). Establish a psychological file system

The database of the students' psychological file system is based on the cooperation of the patient's nursing file system, the evaluation database, the daily life database and the quality training of the nursing staff, group therapy training, etc. The patient care file system based on network information should include data and image information, daily treatment, nursing measurement and incentives. The database helps to fully understand the patient, and if necessary, comprehensively and accurately understand the target of intervention at the first time and help clinical nursing assessors to make accurate judgments quickly. One is to standardize the clinical nursing crisis intervention mechanism of college students and improve the clinical nursing crisis intervention model. The second is to help college students in a state of clinical care crisis relieve acute symptoms, regain their initiative, and reduce heart disease and obstacles after being injured; and help patients in crisis to restore the balance of clinical care as soon as possible to achieve 
harmonious clinical care, or through The crisis has grown. The third is to mobilize various support systems to cope with and solve the factors that cause patients' clinical nursing crisis and reduce the risk of chronic adaptation disorders; the fourth is to carry out research and treatment of patients' clinical nursing crisis intervention and explore the prevention and intervention of patients' clinical nursing crisis suitable for China's national conditions localization model.

b). Carry out systematic psychological health education

Popularizing mental health treatment is an effective way to prevent students' psychological crisis. After years of hard work, the mental health treatment system at all levels has been widely welcomed in mental health treatment training at all levels. On this basis, in order to make the student's psychotherapy cover all aspects of life, including self-awareness, evaluation, social adaptation and development, interpersonal relationships, adjustment, development, treatment and emotional adjustment, emotional sublimation, career planning and preparation, psychological The setbacks and problems on the need to design psychophysiological health treatment and psychological quality training system. Pay attention to the spread of crisis prevention and treatment knowledge to improve the patient's psychological self-healing ability and resistance to adverse factors.

c). Strengthen the cultivation of psychological quality

The student's psychological crisis is mainly due to the fact that minor problems are not properly dealt with properly, and gradually accumulate more and more problems. Psychological quality training can provide effective help to students, improve mental endurance and conflict handling ability. Specifically, psychological quality training is mainly for group counseling, helping students know that they are the soul of the subject, through various relationships between groups, beyond their own boundaries to heal and recognize their inner strength; learn to express their feelings and imagination Strength; Treat yourself to eliminate prejudice and help others develop themselves.

d). Develop various mental problem plans

When formulating various college student' psychological crisis intervention plans: In order to improve the timeliness and effectiveness of psychological intervention, improve the ability of emergency psychological crisis, minimize the damage caused by sudden psychological crisis and adverse consequences, and maintain physical and physical health patient.

\section{Conclusion}

With the development of society and frequent interpersonal communication, the factors that affect college students' mental health are becoming more and more complicated. Therefore, it is urgent to popularize mental health knowledge. For the physiological and psychological characteristics of college students, as well as the more common psychological problems of patients, in addition to popularizing general mental health knowledge, attention should be paid to frustration treatment, love concept treatment, and other aspects.

\section{References}

[1] Yan Wenbo, Dai Shengnan. The system advantage is the magic weapon to win the epidemic prevention and control [EB / OL]. (2020-02-19). Http://theory.people.com.cn/BIG5/n1/ 2020/0219 / c40531-31594828.html.

[2] Jiang Hui. Converting political advantages into epidemic prevention and control advantages [N]. People's Daily, 2020-02-20 (9).

[3] Zhu Zhiping. Research on Social Security Risk Governance of Super Large Cities under the New Situation [J]. Practice and Theory of Special Zones, 2018 (5): 84-89.

[4] Gong Zhigang. Social risk prevention and control and strategic transformation of policing during the historical period [J]. Public Security Research, 2018 (1): 55-77. 